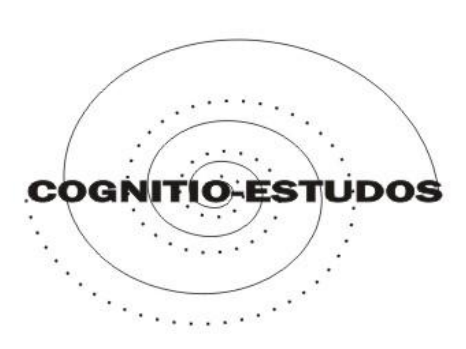

Revista Eletrônica de Filosofia

Philosophy Eletronic Journal

ISSN 1809-8428

São Paulo: Centro de Estudos de Pragmatismo

Programa de Estudos Pós-Graduados em Filosofia

Pontifícia Universidade Católica de São Paulo

Disponível em http://www.pucsp.br/pragmatismo

Vol. 14, no. 2, julho-dezembro, 2017, p.261-277

DOI: $10.23925 / 1809-8428.2017 v 14 i 2 p 93-108$

\title{
O SISTEMA ARISTOTÉLICO DE DEDUÇÃO NATURAL INTERPRETADO NOS GRAFOS EXISTENCIAIS DE C. S. PEIRCE
}

\author{
José Renato Salatiel ${ }^{1}$ \\ Universidade Federal do Espírito Santo (UFES) \\ jrsalatiel@hotmail.com
}

\begin{abstract}
Resumo: A lógica aristotélica tem sido reconstruída em diferentes sistemas formais desde o século passado. Hoje, a interpretação da silogística como um sistema de dedução natural, proposta por Corcoran e Smiley nos anos 1970, é considerada a mais coerente com os Primeiros Analíticos. Por outro lado, a prova de validade dos silogismos categóricos em diagramas remonta aos séculos $18 \mathrm{e}$ 19, com os círculos de Euler e Venn. Pouca atenção foi dada, contudo, aos Grafos Existenciais (GE) de Charles S. Peirce, reconhecidos como um dos mais prolíficos e completos sistemas diagramáticos já inventados. A proposta deste artigo é traduzir o modelo de Corcoran-Smiley para a sintaxe do sistema Beta dos GE, que corresponde à lógica de primeira ordem. Objetiva-se, com isso, elucidar vantagens do sistema diagramático peirciano em relação aos tradicionais de Euler e Venn. Argumentamos que os GE seriam mais adequados para a silogística pelo fato de terem sido concebidos como instrumento de análise do raciocínio dedutivo.
\end{abstract}

Palavras-Chave: Lógica aristotélica. Silogismo. Dedução natural. Diagramas. Grafos Existenciais.

\section{ARISTOTLE'S NATURAL DEDUCTION SYSTEM INTERPRETED INTO C.S. PEIRCE'S EXISTENTIAL GRAPHS}

Abstract: Since the last Century, there have been many attempts to reconstruct Aristotle's logic in terms of formal logic. On the one hand, an interpretation of syllogistic as a natural deduction system was proposed by Corcoran and Smiley in the 1970's. Today, this is interpretation is considered as the most consistent with Prior Analytics. On the other hand, the proof of validity of categorical syllogisms by diagrams goes back to the 18th and 19th Centuries, with the Euler's circles and Venn's diagrams. Nevertheless, logicians have paid little attention to Charles S. Peirce's Existential Graphs, which have been recognized as one of the most prolific and complete diagrammatic logic ever invented. The aim of this paper is to elucidate the advantages of Peircean diagrammatic logic compared to the EulerVenn's traditional systems. I will argue that Existential Graphs provided a more suitable method for studying syllogistic, because they have been created as a tool for the analysis of the deductive reasoning.

Keywords: Aristotle’s logic. Syllogism. Natural deduction. Diagrams. Existential Graphs.

1 O presente trabalho apresenta resultados de pesquisa financiada pelo PNPD/CAPES, no período de 2013-2016, junto ao PPGFIL da UFES. 


\section{Introdução}

A lógica aristotélica, restrita neste trabalho à teoria dos silogismos categoriais (não-modais), tem sido contemporaneamente interpretada em diferentes modelos lógico-matemáticos. No âmbito da lógica simbólica, que compreende sistemas formalizados em linguagem discursiva, duas sistematizações da silogística dominaram o debate no século 20. A primeira, mais sujeita a críticas, foi o modelo axiomático desenvolvido por Jan Łukasiewicz (1957), que interpretava os silogismos como extensivos à lógica proposicional. Posteriormente, John Corcoran e Timothy Smiley (1972-1973) propuseram uma teoria baseada no método de dedução natural, que empregava regras de inferência no lugar de axiomas, e que não pressupunha nenhuma lógica subjacente.

Por outro lado, desde o século 18, os silogismos veem sendo representados, de maneira mais ou menos eficiente, no domínio das chamadas lógicas heterogêneas, que são aquelas que combinam notação simbólica e diagramática (ALLWEIN \& BARWISE, 1996). Os círculos de Euler e os diagramas de Venn são os primeiros sistemas representacionais diagramáticos conhecidos e ainda usados na demonstração de argumentos compostos por proposições categoriais. Pouca atenção foi dada, no entanto, a um dos mais prolíficos e completos sistemas lógicos diagramáticos já inventados, os Grafos Existenciais (doravante, GE) de Charles Sanders Peirce, cujo mérito foi o de ampliar o escopo das linguagens diagramáticas anteriores.

A proposta deste trabalho é fazer uma tradução do modelo de CorcorcanSmiley para a sintaxe diagramática dos GE, objetivando evidenciar as vantagens do sistema diagramático peirciano em relação aos anteriores, de Euler e Venn, no estudo do raciocínio dedutivo e silogístico. Essas vantagens dizem respeito à possibilidade de se fazer uma análise da inferência dedutiva de um modo mais intuitivo e direto, por meio dos diagramas. O ponto é que tal análise, argumentamos, não é possível nos sistemas de Euler e Venn, em razão de eles terem sido concebidos, diferentemente dos GE, como linguagens formais de representação de silogismos válidos, não de demonstração de sua validade por meio de regras de inferência.

Como resultado, endossamos o exame da lógica aristotélica sob o ponto de vista do cálculo de dedução natural, em razão de uma mesma concepção filosófica de lógica ter norteado os trabalhos de Peirce e da chamada tradição modelo-teórica, na qual se inscreve a interpretação de Corcoran. Além disso, propomos os GE como uma alternativa heurístico-pedagógica ao estudo da teoria da dedução de Aristóteles, mediante o uso de diagramas.

No primeiro capítulo apresentamos em detalhes a abordagem da silogística via dedução natural de Corcoran-Smiley, considerada mais fidedigna em relação aos escritos de Aristóteles. Na sequência, examinaremos os sistemas diagramáticos de Euler e Venn, dentro de um recorte histórico que tornará mais compreensível as bases do desenvolvimento ulterior feito por Peirce. No terceiro capítulo tratamos dos GE e propomos uma tradução do modelo de Corcoran-Smiley para sua sintaxe diagramática. Restringimos o presente estudo aos silogismos categóricos, isto é, 
não-modais, e ao conjunto de silogismos que dispensam pressupostos existenciais e cujos modos válidos se circunscrevem às três figuras descritas originalmente por Aristóteles nos capítulos de 1 a 6 dos Primeiros Analíticos.

\section{A estrutura dedutiva dos silogismos segundo Corcoran-Smiley}

No começo dos anos 1970, J. Corcoran (1972) e T. Smiley (1973) elaboraram, independentemente, uma interpretação da lógica aristotélica coerente com os textos dos Primeiros Analíticos e consistente com os parâmetros da moderna lógica matemática. Nessa perspectiva, a silogística não-modal é apresentada como um sistema de dedução natural, em oposição à sua reconstrução como um sistema axiomático de cálculo proposicional feita previamente por J. Łukasiewicz (1957)2.

Métodos de dedução natural foram elaborados independentemente por Stanisław Jaśkowski e Gerhard Gentzen nos anos 1930³. Diferentemente dos sistemas axiomáticos da tradição de Frege-Russell, a dedução natural não usa, de modo geral, axiomas, mas regras de inferências para a demonstração de teoremas. Essas regras instruem a manipulação de símbolos lógicos que permitem passar de uma sentença a outra, acompanhando o raciocínio dedutivo passo a passo, das premissas à conclusão.

Segundo Corcoran (1972, 1974), a silogística aristotélica inclui um sistema de dedução natural, sendo assim, é uma teoria da demonstração de argumentos válidos, entendidos como aqueles em que a conclusão é uma consequência lógica das premissas. Contrário a Łukasiewicz, para quem o silogismo era uma implicação material, Corcoran e Smiley o interpretam como uma dedução, noção mais compatível com a conceituação de Aristóteles, de que silogismos "[...] (ou dedução) é um argumento no qual, certas coisas sendo supostas, algo diferente delas decorre necessariamente" (Pr. An. I 1, 24b 18-22).

A uma dedução válida Aristóteles chama silogismo perfeito, ao passo que um silogismo imperfeito é aquele em que a cadeia de raciocínio que leva das premissas à conclusão não é evidente, sendo necessário adicionar mais proposições de modo a obter a sua demonstração (Pr. An. I 1, 24b 20-25). Assim, deduções indiretas (os silogismos imperfeitos) seriam obtidas pela aplicação sistemática de regras de inferência, que incluem os modos silogísticos da primeira figura, os chamados silogismos perfeitos. O modelo de Corcoran-Smiley, portanto, considera esse processo de "perfectibilização" dos silogismos como um processo dedutivo.

O sistema formal silogístico, nesse aspecto, tem os seguintes elementos formais em uma linguagem $L$ :

\section{Vocabulário:}

\footnotetext{
2 O trabalho de Łukasiewicz foi publicado pela primeira vez em 1951 e exerceu considerável influência entre os lógicos nas duas décadas seguintes, a despeito de críticas, até o surgimento de novas técnicas em lógica e o advento das interpretações de Corcoran (1972), Smiley (1973) e Lear (1980).

3 Tais métodos foram propostos por Łukasiewicz em 1926 e publicados por Jaśkowski, em 1934, no artigo intitulado "Sobre as regras de suposição na lógica formal" (On the rules of suppositions in formal logic), e por Gentzen em "Investigações sobre dedução lógica" (Untersuchungen über das logische Schließen), publicado originalmente em duas partes, em 1934 e 1935.
} 
(i) Quatro constantes lógicas, que designamos pelas letras maiúsculas $A$, $\mathrm{E}$, I e O, representando expressões quantificadas;

(ii) Um conjunto infinito $V$ de constantes não lógicas $\left\{\alpha^{\prime}, \alpha ”, \alpha ” \ldots\right.$ etc $\}$, representando termos categóricos.

2. Regras de formação:

(i) Uma fórmula em L é composta anexando a uma constante quantificada dois termos categóricos distintos, obtendo assim quatro proposições categóricas: Aab ("Todo A é B"), Eab ("Nenhum A é B"), lab ("Algum A é B") e Oab ("Algum A não é B").

(ii) Nada mais é uma fórmula.

Definição (1): Um argumento é um par ordenado $(P, c)$, onde "P" é um conjunto de sentenças, chamadas premissas ${ }^{4}$, e "c" é uma sentença única, chamada conclusão.

3. Semântica de L:

(i) Axy é verdadeiro se, e somente se, todo $x$ está incluído em y $(x \subseteq Y)$, e falso em caso contrário;

(ii) Exy é verdadeiro se, e somente se, nenhum $x$ está incluído em y $(x \cap y$ $=\varnothing$ ), e falso em caso contrário;

(iii) Ixy é verdadeiro se, e somente se, algum $x$ não está incluído em y $(x \cap$ $y \neq \varnothing$ ), e falso em caso contrário; e

(iv) Oxy é verdadeiro se, e somente se, algum $x$ não está incluído em y ( $\mathrm{x}$ $\not \subset \mathrm{Y})$, e falso em caso contrário.

Com base nessas interpretações, podemos definir também os seguintes pares contraditórios:

Definição (2): São pares contraditórios ${ }^{5}$ as seguintes fórmulas:

(i) $\quad c(d)=$ Axy sse $d=O x y$

(ii) $\quad c(d)=$ Exy sse $d=I x y$

(iii) $\quad c(d)=$ Ixy sse $d=$ Exy

(iv) $\quad c(d)=$ Oxy sse $d=A x y$

4. Regras de inferência:

(i) Regra dos silogismos perfeitos:

(SP1) Azy, Axz $\vDash A x y$.

(SP2) Ezy, Axz $\vDash E x y$.

(SP3) Azy, Ixz $\vDash I x y$.

(SP4) Ezy, Ixz $\vDash$ Oxy.

(ii) Regras de conversão

Conversão simples:

\footnotetext{
${ }^{4}$ Aristóteles afirma que um silogismo deve ter ao menos duas premissas, mas não descarta que possa ter mais de duas premissas. Uma discussão a respeito disso pode ser encontrada no cap. 2 de LEAR, 1980.

${ }^{5}$ Duas proposições são contraditórias se, e somente se, não puderem ser ambas verdadeiras e nem ambas falsas.
} 
(Convesão-E) Exy $\vDash$ Eyx;

(Conversão -l) Ixy $\vDash$ lyx.

Conversão por limitação:

(Conversão-A) Axy ₹ lyx.

Silogismos perfeitos, conforme dito, são aqueles argumentos dedutivos válidos nos quais a conclusão segue-se imediatamente de suas premissas, diferente dos silogismos imperfeitos, que precisam ser "perfectibilizados". Segundo Aristóteles, isso pode ser feito de dois modos: por meio das regras de inferências (regras de primeira figura e de conversão), ou indiretamente, pelo princípio de reductio ad absurdum (ou prova por contradição). Essa prova consiste em supor uma conclusão contraditória àquela pretendida, i.e., $\alpha$, e a partir dela derivar uma contradição, $\mathrm{p} \wedge \sim \mathrm{p}$, mostrando, assim, que o contraditório à suposição, $\alpha$, é verdadeiro. Cesare:

Vejamos o seguinte exemplo do silogismo da segunda figura, conhecido como

(i) Eyz, Axz $\vdash$ Exy
1. Eyz
Premissa 1
2. $A x z$
Premissa 2
3. Ezy
1, conversão-E
4. Axz
2, hipótese
5. Exy
3,4 SP2.

No lado esquerdo numeramos os passos da dedução, seguido pelas fórmulas; no lado direito inserimos a justificativa para cada passo do processo inferencial. Após inserir as duas premissas do argumento, aplicamos a regra de conversão-E à premissa (1); em seguida, usamos a premissa (2) como hipótese e aplicamos a regra (3) do silogismo perfeito, obtendo, assim, a conclusão do argumento. "indireta":

O segundo exemplo, também da segunda figura, Baroco, é uma dedução

(ii) Ayz, Oxz $\vdash$ Oxy
1. Ayz
Premissa 1
2. $O x z$
Premissa 2
3. Axy
RAA
4. Ayz
1, hipótese
5. Axz
3,4 SP1
6. $\mathrm{Oxz}$
2, premissa

Nesse silogismo, aplica-se a regra de redução ao absurdo, postulando (linha 3) uma conclusão contraditória àquela pretendida $(c(d)=A x y$ sse $d=O x y)$. Usando a regra (1) do silogismo perfeito, obtemos Axz (linha 5), que é contraditória à premissa (2), Oxz. Conclui-se que a suposição Axy (linha 3) levou a uma impossibilidade; portanto, o contraditório é verdadeiro (Oxy). 


\section{Os sistemas diagramáticos de Euler e Venn}

Diagramas lógicos são figuras geométricas bidimensionais dotadas de relação isomórfica com certas estruturas lógicas (GARDNER, 1958, p. 28). Eles têm sido usados para representar o processo inferencial dedutivo desde Euclides, com destaque para seu apelo heurístico na obtenção da prova. Mas é consenso entre os historiadores da lógica que o matemático suíço Leonhard Euler foi o introdutor de diagramas para a representação e análise de silogismos, em uma série de manuscritos datados de $1761^{6}$.

Nos círculos de Euler, proposições universais são apresentadas de um modo intuitivo: dois círculos, um contendo o outro, representam a afirmação universal, enquanto dois círculos separados, a negação universal. Assim, o seguinte argumento pode ser facilmente visualizado:

Nenhum C é B [P1],

Todo A é B [P2],

Portanto, nenhum A é C [C].

P1

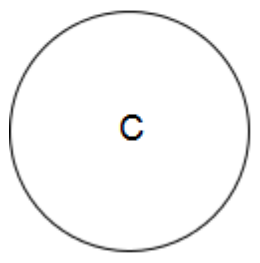

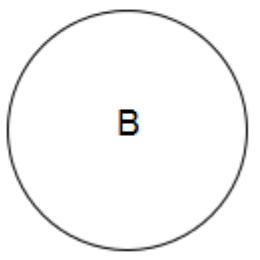

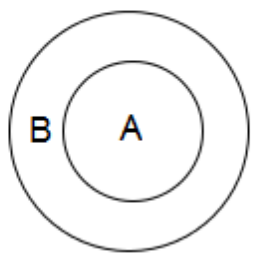

C

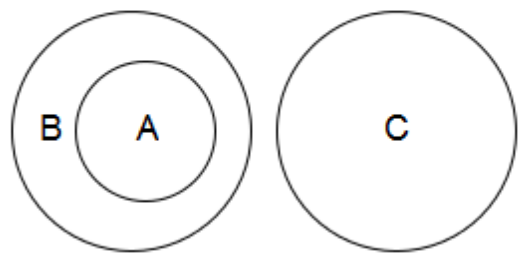

Figura 1

A conclusão do silogismo é obtida unificando-se as premissas do argumento, de modo a extrair as informações necessárias para a sua demonstração. É uma manipulação intuitiva que dispensa regras de inferência.

O primeiro problema com os diagramas de Euler surge ao lidar com proposições existenciais, pois a representação pode gerar ambiguidade. Considerese o seguinte exemplo:

Todo C é B [P1],

Algum A não é $B$ [P2],

Portanto, algum $A$ não é $C$ [C]

P1

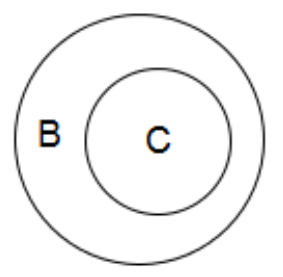

P2

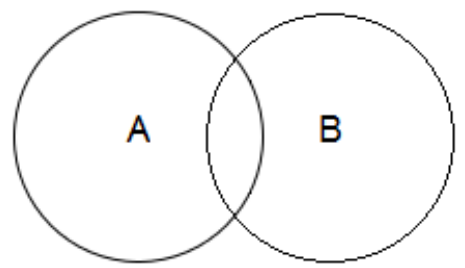

C

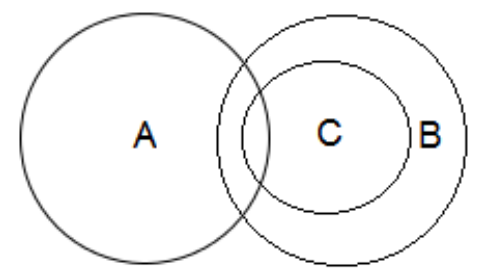

${ }_{6}^{6}$ Os círculos de Euler apareceram pela primeira vez em sete cartas, em 1761, publicadas como Lettres à une princess d'Allemagne, em 1772. 
Figura 2

A segunda premissa, particular negativa, é representada com dois círculos sobrepostos nos quais a posição ocupada pelas letras adquirem relevância, pois a leitura deve ser "Algum A não é B", não "Algum B não é A". No caso de uma proposição particular afirmativa, a letra " $A$ " seria inserida na região correspondente à intersecção entre os círculos. Isso dificultaria a inserção das informações contidas nas premissas em um único diagrama, representando a conclusão?

A vantagem do sistema diagramático do matemático inglês John Venn, apresentado em Symbolic Logic (1881), é justamente possibilitar que as relações possíveis sejam representadas em um único diagrama. Para isso ele insere um novo dispositivo sintático, o sombreamento, que representa negação lógica. Com isso, ao mesmo tempo em que sofistica a linguagem diagramática em relação à Euler, tornase defasada no aspecto intuitivo, pois a semântica se torna indireta, ou seja, a prova requer um conhecimento prévio do significado das áreas sombreadas. Observe-se o seguinte exemplo:

Nenhum C é B [P1],

Todo A é B [P2],

Portanto, nenhum A é C [C].
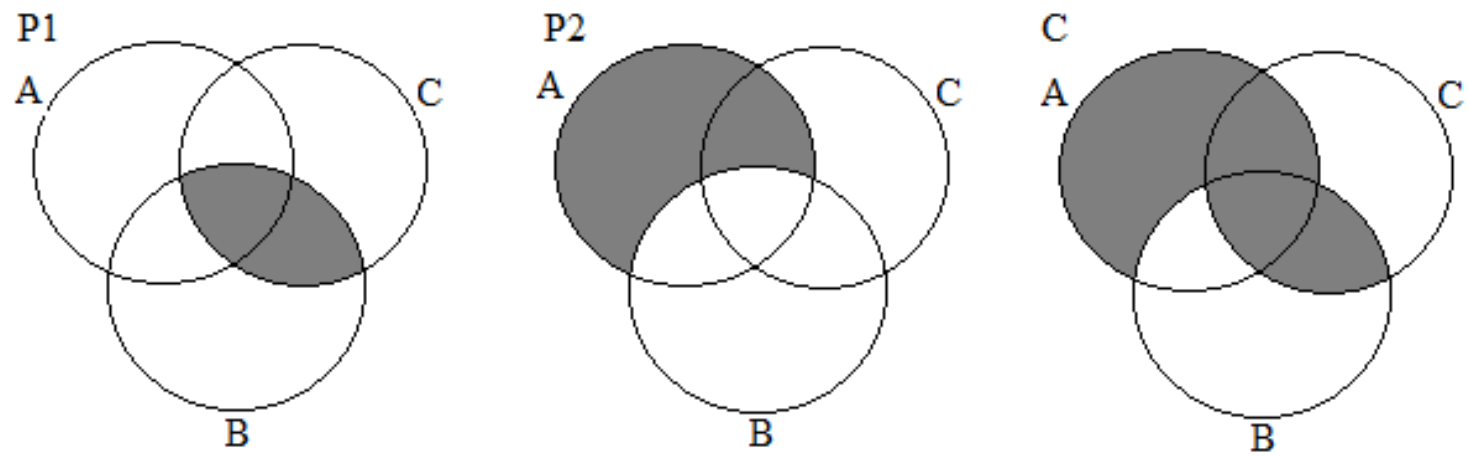

Figura 3

Aqui a manipulação é realizada não unificando os diagramas das premissas, mas sobrepondo-os, de forma a derivar a conclusão do argumento. A validade do silogismo é assegurada porque, no diagrama da conclusão, a área a ser sombreada já está sombreada, a partir da sobreposição das premissas.

O sistema de Venn, entretanto, também falhava em representar proposições existenciais (o sombreamento só funciona na expressão de universalidade). Para resolver isso Peirce introduziu o símbolo " $x$ " no sistema (CP 4.359) ${ }^{8}$. Desse modo, 0 seguinte silogismo pode ser diagramado:

\footnotetext{
7 Para uma análise dos defeitos envolvendo ambiguidade na interpretação dos círculos de Euler e nos diagramas de Venn, cf. SHIN, LEMON \& MUMMA, 2013.

8 Peirce propôs, na verdade, a inserção de três dispositivos sintáticos no sistema lógico de Venn, de modo a permitir que expressasse proposições existenciais e disjuntivas. Além do " $x$ " para conteúdo existencial, o símbolo "o" para substituir o sombreamento, e "-", conectando os dois primeiros, para representar disjunção. Essa estratégia, contudo, compromete a intuitividade dos diagramas, que é a maior característica dos sistemas Euler-Venn.
} 
Todo C é B [P1],

Algum A não é B [P2],

Portanto, algum $A$ não é $C[C]$
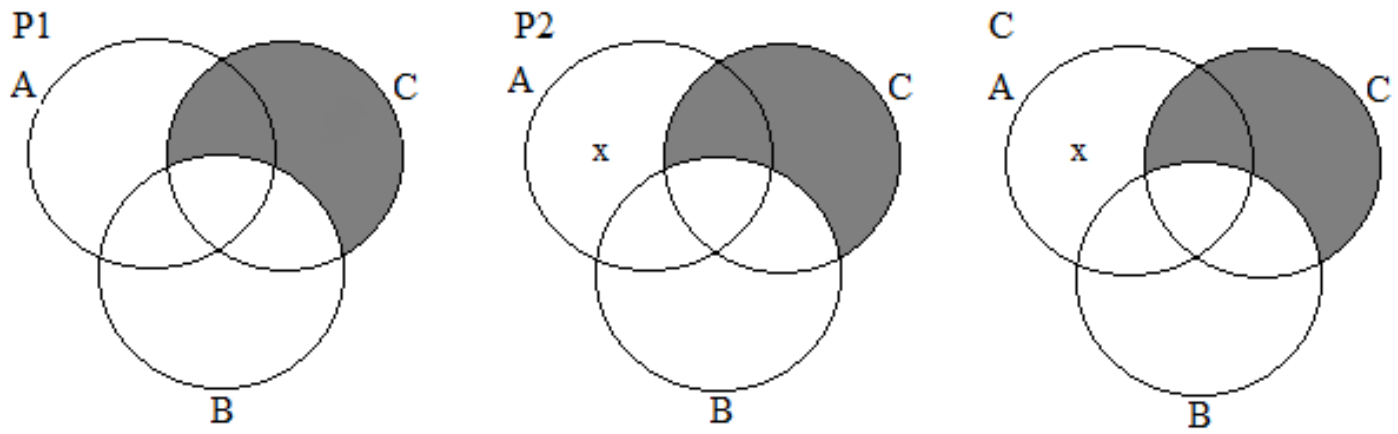

Figura 4

Observe que o " $x$ " na região representa um existente em "A" que não é " $B$ " $e$, como é a mesma região a ser inserido o " $\mathrm{x}$ " para algum " $A$ " que não é " $C$ ", a conclusão já está dada nas premissas, e o argumento, assim, é válido.

A questão que queremos destacar aqui é que esses sistemas diagramáticos clássicos, ainda que eficazes para a representação de silogismos não-modais, falham em apreender a teoria da dedução aristotélica, ou seja, em fornecer uma análise do raciocínio dedutivo conforme apresentada no cálculo de dedução natural. A razão disso é que eles não foram concebidos como sistemas de demonstração, isto é, um procedimento para determinar a validade de argumentos por meio de uma cadeia de raciocínios que evidenciem os passos dedutivos e mostrem que a conclusão é uma consequência lógica das premissas. Ambos os sistemas, de Euler e Venn, não foram criados com regras rigorosas que prevenissem a ambiguidade na manipulação de informações e, ao mesmo tempo, explicitassem o processo dedutivo.

Nos anos 1990 foram realizados trabalhos importantes no sentido de adaptar essas linguagens diagramáticas tradicionais ao rigor e precisão da lógica de primeira ordem, dotanda-as de sintaxe e semântica formais, bem como definindo propriedades lógicas como consequência, completude de decidibilidade (cf. STAPLETON, 2005). São exemplos os estudos de caso de Shin (1994-1996) e Hammer \& Danner (1996) sobre os diagramas de Venn, modificando sua notação de modo a aumentar a expressividade sem perda da simplicidade visual. O desafio, nessas pesquisas, é prover os sistemas Euler-Venn de uma formalização que atenda aos padrões da lógica moderna sem que, com isso, a complexidade acabe por comprometer o caráter intuitivo dos diagramas na representação do raciocínio dedutivo, justamente o principal benefício auferido pela linguagem diagramática à lógica.

\section{Grafos Existenciais de Peirce}

Discussões de Peirce a respeito dos limites dos sistemas de Euler e, principalmente, Venn (CP 4.362), o levaram a desenvolver seu próprio sistema 
gráfico para suprir uma falha insuperável nesses dois que o precederam: a incapacidade de representar fórmulas quantificadas e relações, em outras palavras, em ajustar-se ao que chamamos hoje de lógica de predicados. Para isso, Peirce incorporou a uma notação diagramática um conjunto de regras de transformação, décadas antes do advento dos cálculos de dedução natural, o que faz dele também um precursor dessa técnica.

Os GE dividem-se em três sistemas complementares, o Alfa, que corresponde à lógica proposicional; o Beta, que parcialmente corresponde a uma lógica de predicados com identidade; e o Gama, que não chegou a completar e que seria, para alguns comentadores, uma lógica modal (cf. ROBERTS, 1973; SHIN, 2002; e SOWA, 2011).

Para a silogística utilizaremos a sintaxe do sistema Beta, adequada para representar as proposições categóricas, e traduziremos sua semântica para a silogística. Com respeito à prova, adotaremos as regras do modelo de CorcoranSmiley, definidas a partir dos Primeiros Analíticos, não obstante os GE terem seu próprio conjunto de regras de transformação.

Chamemos $L_{\beta}$ à linguagem do sistema Beta para a lógica silogística e designemos os símbolos metalógicos " $\alpha$ " e " $\beta$ " para qualquer fórmula nesse sistema. Definiremos então do seguinte modo a linguagem formal $L_{\beta}$ :

1. Vocabulário:

(i) Letras maiúsculas "A", "B" e "C" representando constantes predicativas;

(ii) Círculos ao redor das letras, ou "corte", representam a negação lógica do conteúdo do grafo; e

(iii) Linhas conectando as letras, ou "linhas de identidade", denotam a existência de um indivíduo qualquer no universo discursivo.

Essas linhas de identidade são a primeira inovação em sistemas gráficos, em relação aos de Euler e Venn, pois elas inserem um dispositivo visual para identificar os quantificadores nas fórmulas, algo ausente em sistemas formais diagramáticos até então ${ }^{9}$. Por meio dessa sintaxe simples podemos representar (figura 5) as quatro proposições aristotélicas, "Todo A é B" (I), "Nenhum A é B" (II), "Algum A é B" (III) e "Algum A não é B" (IV), do seguinte modo:

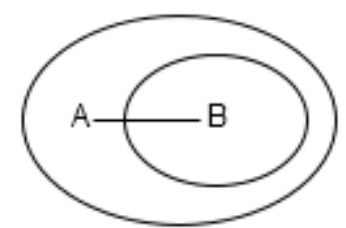

(I)

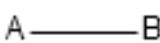

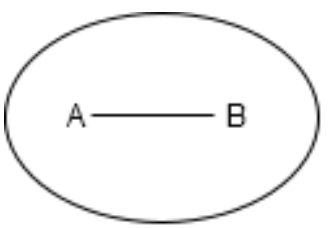

(II)

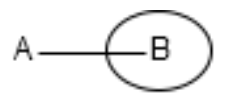

9 Todos os grafos do sistema Beta podem ser lidos como quantificadores existenciais, que se diferenciam dos universais exclusivamente pela observação dos elementos visuais dos diagramas. Segundo Peirce, "[...] qualquer linha de identidade cuja maior parte é fechada em [cortes] pares se refere a algum, e qualquer outra cuja maior parte é fechada em [cortes] ímpares refere-se a todo" (CP 4.458). A seguir explicitamos a diferença entre cortes "em pares" e "em ímpares". 
(III)

Figura 5

A proposição particular afirmativa (III) é representada como uma linha conectando os termos "A" e "B". O corte é a negação do conteúdo do grafo, então, temos apenas que circular completamente o grafo de uma particular afirmativa para obter uma universal negativa (II). E circulando somente a letra "B" do mesmo grafo o resultado é "Algum A não é B" (IV).

A proposição universal afirmativa (I) requer um pouco mais de atenção em sua leitura. $\mathrm{Na}$ semântica de Peirce para os $\mathrm{GE}^{10}$, lemos um grafo de fora para dentro. Portanto, lemos o primeiro grafo como "Não é verdadeiro que $A$ e não B", ou "Todo A é B" - em linguagem de lógica de predicados, $\neg \exists x(A x \wedge \neg B x)$ ou $\forall x(A x \rightarrow$ $\mathrm{Bx})$.

2. Regras de formação:

(i) Um grafo com duas letras predicativas distintas e unidas por uma linha de identidade é uma fórmula em $L_{\beta}$;

(ii) Um círculo em torno de uma ou de duas letras predicativas (corte), fechando parcialmente ou completamente o grafo, é uma fórmula em $L_{\beta}$;

(iii) Nada mais é uma fórmula em $L_{\beta}$.

3. Semântica de $L_{\beta}$ :

(i) Um grafo com duas constantes " $\alpha$ " e " $\beta$ " unidas por uma linha de identidade traduz-se como "Algum a é $\beta$ ";

(ii) Um grafo com duas constantes " $\alpha$ " e " $\beta$ " unidas por uma linha de identidade e na qual a constante " $\beta$ " é fechada por um corte, traduzse como "Algum $\alpha$ não é $\beta$ ";

(iii) Um grafo com duas constantes " $\alpha$ " e " $\beta$ " unidas por uma linha de identidade e completamente fechado por um corte traduz-se como "Nenhum $\alpha$ é $\beta$ ";

(iv) Um grafo com duas constantes " $\alpha$ " e "B" unidas por uma linha de identidade em que " $\beta$ " é fechada por um corte e o grafo inteiro é fechado por outro corte, traduz-se como "Todo $\alpha$ é $\beta$ ".

Essas definições semânticas permitem ainda verificar as contradições lógicas entre pares opostos de proposições apenas observando os diagramas ${ }^{11}$.

\footnotetext{
10 Há diferentes propostas de leitura dos Grafos Existenciais feitas pelos comentadores, condizentes com o nível de complexidade requerido pelos diagramas (para uma discussão a esse respeito, cf. cap. 5 de SHIN, 2002). O sistema Beta, por exemplo, foi desenvolvido para lidar com quantificações múltiplas com identidade, o que exigiria uma descrição mais rigorosa de um protocolo de leitura de grafos. Para os propósitos da silogística, no entanto, restrita às quatro proposições categóricas, será suficiente esse método simples que Peirce chamou de endoporêutico, que consiste em começar a leitura com os cortes (de fora) e continuar para o interior do grafo, seguindo as convenções estabelecidas para a linguagem. (ROBERTS, 1973, p. 39, n. 13).

11 Interpretando o quadrado clássico das oposições nessa linguagem diagramática dos GE, evidencia-se visualmente os pares contraditórios, mas não as relações de contrariedade e
} 


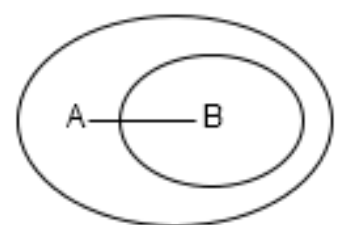

(I)

Figura 6

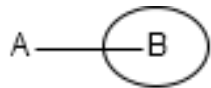

(II)

Assim, no primeiro grafo (figura 6, I), "Todo A é B", o termo "A" está em uma área que chamaremos "área-l" ou "negativa" (ou, como diz Peirce, "fechada em [cortes] ímpares"), e o termo "B" está em uma área "área-P" ou "positiva" (ou "fechada em [cortes] pares"). Essa relação observada é exatamente o oposto em "Algum A não é B" (II), no qual o termo "A" está em uma área-P (sem cortes) e o termo "B" em uma área-I (fechado em um corte). Portanto, essas duas proposições são obviamente contraditórias. A mesma relação pode ser inferida nas outras duas proposições categóricas, "Nenhum A é B" (III) e "Algum A é B" (IV) (figura 7):

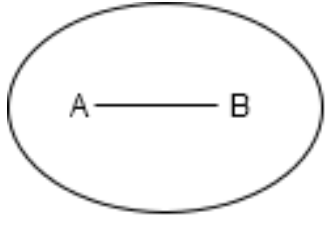

(III)

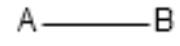

(IV)

Figura 7

Definição (3): São pares contraditórios as seguintes fórmulas:

(i) Seja d um grafo em que " $\alpha$ " estiver inscrita em uma área-I (ou área-P) e " $\beta$ " em uma área-P (ou área-I), estão, em $c(d)$ " $\alpha$ " é inscrita em uma área-P (ou área-I) e " $\beta$ " em uma área-I (ou área-P).

Por fim, a segunda inovação de Peirce nos sistemas diagramáticos é a adoção de regras de inferência, ausente nos sistemas de Euler e de Vem. São essas regras que tornam os GE um sistema de dedução natural, próximo àqueles propostos por Gentzen e Jaśkowski (na verdade, Peirce os antecipam, cf. ROBERTS, 1973, p. 121; e SHIN, 2002, pp. 80-83), e que permitem interpretar a silogística de um modo mais próximo de suas leituras modernas ${ }^{12}$.

Porém, para a tarefa a qual nos propomos aqui, a de reconstrução do cálculo de silogismos categóricos, serão suficientes as regras definidas no modelo Corcoran-Smiley (capítulo 1), bastando para isso sua tradução no vocabulário do sistema Beta dos GE.

\section{Regras de inferência:}

subcontrariedade, que exigem a suposição de conteúdo existencial. Tal interpretação é coerente com a da lógica moderna, que reduz o quadrado de oposições às relações de contraditoriedade.

${ }^{12}$ As regras de transformação dos GE são similares àquelas dos sistemas de dedução natural, com pares de regras para inserção e apagamento de grafo (uma síntese dessas regras pode ser verificada em ROBERTS, 1973, pp. 41-43; SHIN, 2002, pp. 136-137; e SOWA, 2011 pp. 56-59). 
(i) Regra dos silogismos perfeitos:

(SP1) Abc, Aab $\vDash$ Aac.

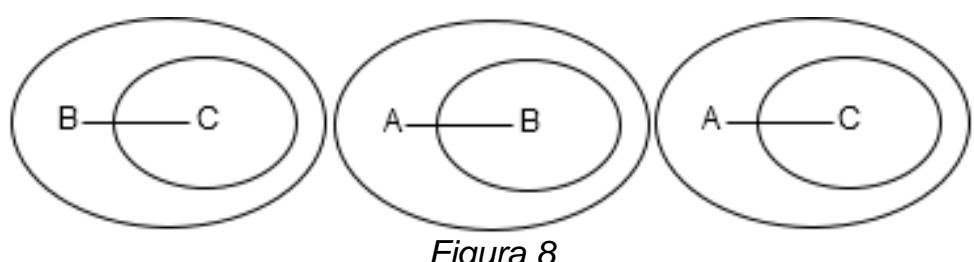

(SP2) Ebc, Aab ₹ Eac.

Figura 8

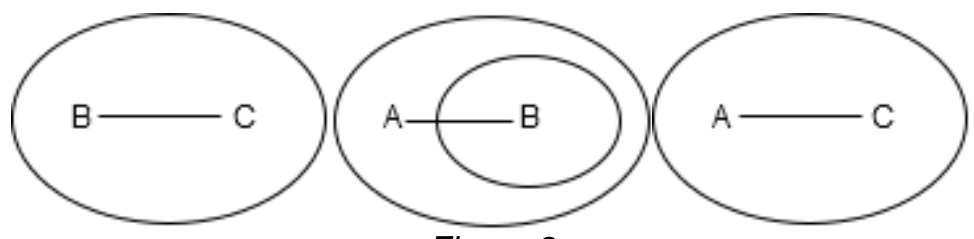

Figura 9

(SP3) Abc, lab $\vDash l a c$.

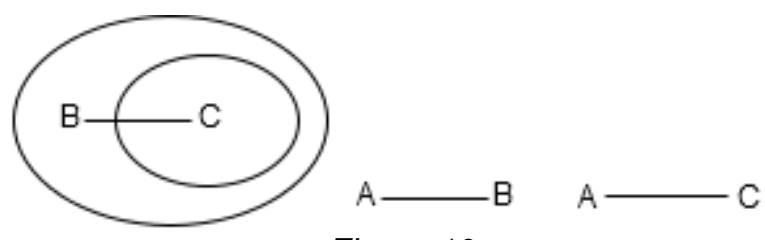

Figura 10

(SP4) Ebc, lab $\vDash O a c$.

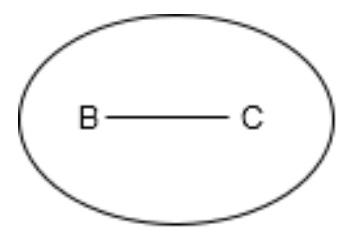

A-B

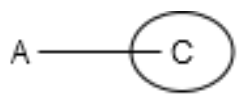

Figura 11

(ii) Regras de conversão:

Para as regras de conversão simples, apenas trocamos as posições dos símbolos de constantes de predicados nos grafos: Eab $\vDash E b a$; lab $\vDash l b a$. Para a conversão por limitação, além de trocar as posições dos termos $(A a b \vDash l b a)$, eliminamos os cortes, conforme mostrado abaixo nos diagramas abaixo:

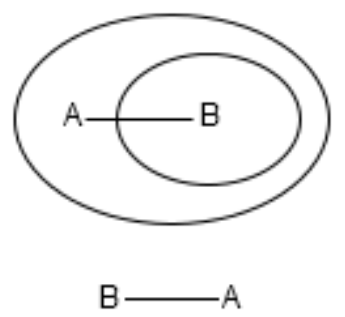

Figura 12

Esse tipo de conversão, entretanto, não é permitido no sistema Beta, pois as regras de transformação do sistema (e essa é a única exceção em que as citamos neste artigo) possibilitam a eliminação de um duplo corte, o que não é o caso do 
grafo da figura 12, em que há dois cortes (um deles cercando o termo "A" e o outro, o termo "B"), não um duplo (cercando ambos os termos). De todo modo, a regra de conversão por limitação só seria necessária para o cálculo se considerássemos a extensão dos modos silogísticos válidos para incluir aqueles em que é admitida a suposição existencial ${ }^{13}$. Para este trabalho, conforme dito na introdução, consideramos apenas os modos silogísticos válidos tradicionais em Aristóteles.

Seguem-se os exemplos de demonstrações dos modos silogísticos Cesare (EAE) e Baroco (AOO) da segunda figura:

(i) Cesare (EAE)

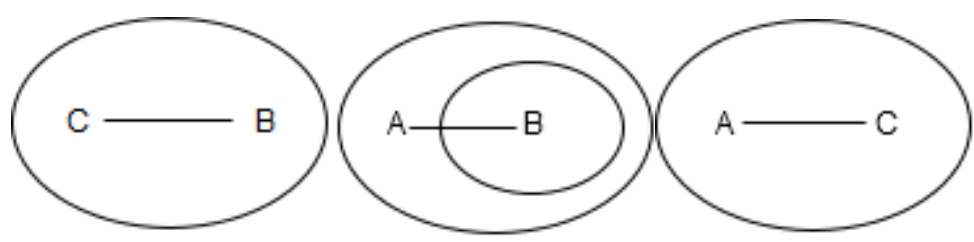

Ecb, Aab $\vdash$ Eac (Cesare)

1.

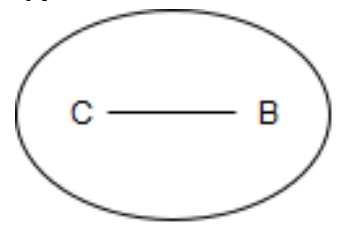

Premissa 1

2.

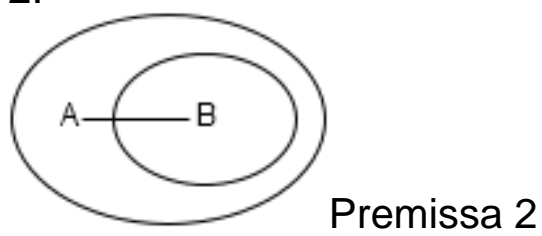

3.

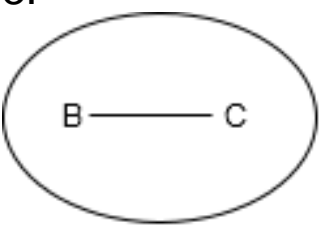

1 , conversão-E

4.

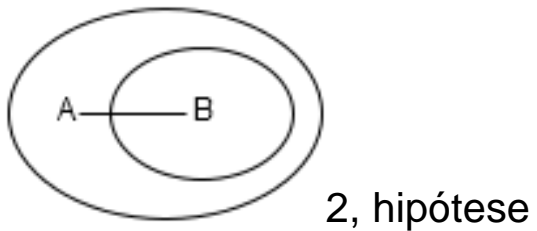

5.

${ }^{13}$ Essa hipótese consiste em adicionar uma premissa ao silogismo que infira a existência de um de seus termos, de modo a validar o argumento (cf. STRAWSON, 1952, p. 164-165). 


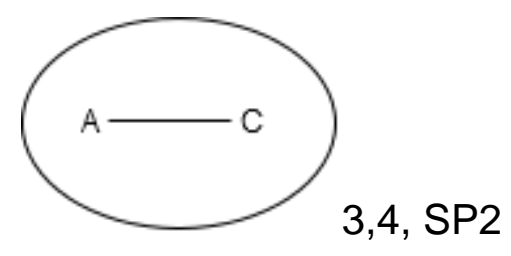

Figura 13

(ii) Baroco (AOO)

1.
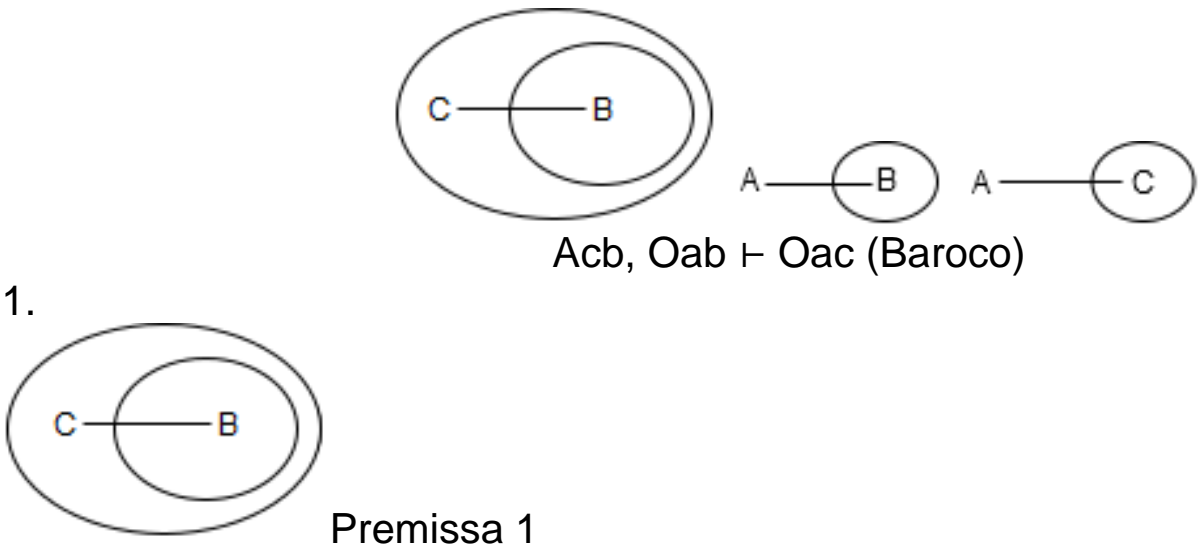

2.

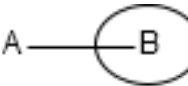

Premissa 2

3.

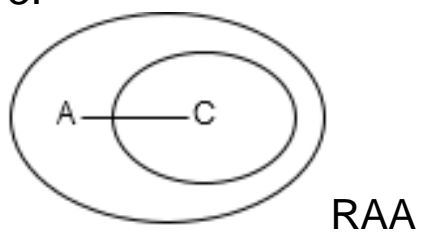

4.

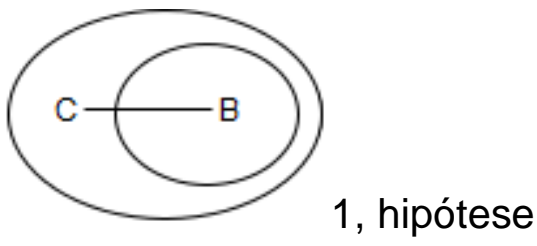

5.

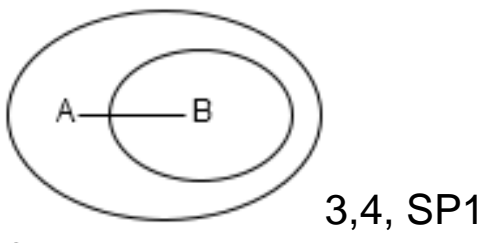

6.

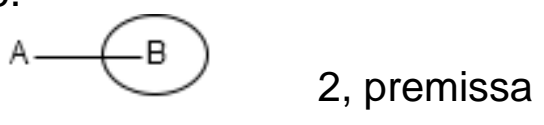

Figura 14

Os grafos do sistema Beta permitem, conforme mostrado nos exemplos (figuras 13 e 14), a demonstração de silogismos usando o mesmo aparato dedutivo 
da lógica aristotélica, reconstruída como cálculo de dedução natural e interpretado em linguagem diagramática. Isso não seria possível no vocabulário de Euler-Venn por esses terem sido criados como mecanismos de representação de silogismos, não de análise de raciocínio dedutivo. A inserção de mais dispositivos simbólicosintáticos nesses sistemas diagramáticos, por outro lado, traz sempre o risco de, ao lado do ganho de expressividade, a perda da propriedade intuitiva. Nesse sentido, a solução de Peirce parece oferecer vantagens a ambos os polos da questão ${ }^{14}$.

\section{Conclusões}

A comparação da linguagem diagramática de Peirce com os círculos de Euler e Venn apontam para uma vantagem do primeiro em relação aos outros dois: os GE referendam uma leitura da lógica aristotélica como um sistema de dedução natural, sem que para isso seja preciso complexificar o sistema com novos elementos sintático-formais ou mesmo regras de inferência adicionais, bastando apenas fazer a tradução de uma sintaxe para a outra.

Não obstante termos nos limitado aos exemplos de duas figuras silogísticas ao longo deste texto, convém reforçar que a validade de todos os silogismos nãomodais trabalhados por Aristóteles nos capítulos de 1 a 6 dos Primeiros Analíticos podem ser facilmente demonstrados pelo mesmo processo. Sugerimos ainda que os GE podem servir, nesse sentido, como uma interessante ferramenta didática no ensino da silogística ${ }^{15}$.

Há, por fim, um contexto filosófico a ser discutido na conclusão desse trabalho, pois ele elucida a relação isomórfica entre o modelo Corcoran-Smiley e os GE de Peirce. Refiro-me à divisão entre duas tradições da lógica definidas por Jean Van Heijenoort (1967), com base em Frege, entre lógica entendida como (i) linguagem universal (língua characterica) e lógica como (ii) cálculo (calculus ratiocinator). Em linhas gerais, no primeiro caso (i), o conceito central da lógica é o de "verdade lógica", daí o emprego de axiomas na dedução de teoremas; no segundo (ii) a ênfase é o conceito de "consequência lógica", portanto o uso de métodos de análise do processo dedutivo, como o cálculo de dedução natural. À primeira tradição alinham-se nomes como Frege, Russel e o "primeiro" Wittgenstein, enquanto à segunda, Gentzen, Tarski e Peirce (HINTIKKA, 1997).

Segundo Łukasiewicz (1957), a lógica aristotélica é um sistema de proposições organizadas em um modelo formal axiomático, portanto, perfila-se à tradição da linguagem universal. Na interpretação de Corcoran (1972), Smiley (1973) e Lear (1980), ao contrário, Aristóteles se preocupava com o estudo da consequência lógica em silogismos válidos, por isso a lógica apresentada nos Primeiros Analíticos é um conjunto de regras de dedução que se configura como uma lógica subjacente às ciências axiomáticas (FILLON, 2007). Nesse sentido, justificam-se os isomorfismos observados entre as interpretações desses pesquisadores e os estudos de Peirce sobre diagramas.

\footnotetext{
14 Outra proposta interessante, em direção contrária, de simplificação da notação dos diagramas de Venn para a representação de silogismos, foi explorada por SAUTTER, 2010.

${ }^{15}$ Mesmo que, é importante notar, que os GE não se restrinjam às proposições categóricas, pois o objetivo de Peirce, conforme dissemos anteriormente, era expressar teoremas da hoje chamada lógica de primeira ordem.
} 


\section{Referências}

ALLWEIN, Gerard and BARWISE, Jon. Logical reasoning with diagrams. New York and Oxford: Oxford University Press, 1996.

ARISTOTLE. Prior analytics. Robin Smith (trans. e intro.). Indianapolis/Cambridge: Hackett Publishing Company, 1989.

CORCORAN, John. Completeness of an ancient logic, The Journal of Symbolic Logic, vol. 37, n. 4, Dec. 1972, pp. 696-702.

Aristotle's natural deduction system. In: Ancient Logic and its modern interpretation. CORCORAN, J. (ed.). Dordrecht-Holland: D. Reidel Publishing Company, 1974, pp. 85-131.

FILLION, Nicolas. Two accounts of Aristotle's logic: a comparison of Lukasiewicz's and Corcoran-Smiley's reconstructions. In: Seminar on Aristotelian Logic, Faculty of Arts the University of Western Ontario, April 16, 2007.

GARDNER, M. Logic machines and diagrams. McGraw-Hill: New York, 1958.

HAMMER, Eric and DANNER, Norman. Towards a model theory of Venn diagrams. In: Logical reasoning with diagrams. ALLWEIN, Gerard and BARWISE, Jon (ed.). New York/ Oxford: Oxford University Press, 1996, pp. 109-127.

HINTIKKA, Jaakko. The place of Peirce in the history of logical theory. In: The rule of reason: the philosophy of Charles Sanders Peirce. BRUNNING, Jacqueline and FORSTER, Paul (eds.). Toronto/ Buffalo/ London: University of Toronto Press, 1997, pp. 13-33.

LEAR, Jonathan. Aristotle and logical theory. Cambridge: Cambridge University Press, 1980.

ŁUKASIEWICZ, Jan. Aristotle's syllogistic from the standpoint of modern formal logic. $2^{-}$ed. Oxford: Clarendon Press, 1957.

PEIRCE, Charles Sanders. Collected papers. 8 vols. HARTSHORNE, Charles; HEISS, Paul e BURKS, Arthur (eds.). Cambridge: Harvard University Press, 19311958. [Citado como CP seguido do número do volume e parágrafo.]

ROBERTS, Don. The existential graphs of Charles S. Peirce. The Hague: Mouton \& Co, 1973.

SAUTTER, Frank Thomas. A essência do silogismo, Cognitio: Revista de Filosofia, vol. 11, n. 2, julho-dezembro 2010, pp. 316-329.

SHIN, Sun-Joo. The logical status of diagrams. Cambridge: Cambridge University Press, 1994.

Situation-theoretic account of valid reasoning with Venn diagrams. In: Logical reasoning with diagrams. ALLWEIN, Gerard and BARWISE. Jon (ed.). New York/ Oxford: Oxford University Press, 1996, pp. 81-108.

The iconic logic of Peirce's graphs. Cambridge: MIT Press, 2002. 
LEMON, Oliver \& MUMMA, John. Diagrams. In: Stanford Encyclopedia of Philosophy, 2013. Disponível em: http://plato.stanford.edu/entries/diagrams/. Acesso em: 15 jul. 2016.

SMILEY, T. J. What is a syllogism? Journal of Philosophical Logic, vol. 2, n. 1, Jan. 1973, pp. 136-154.

SOWA, John F. Peirce's tutorial on existential graphs, Semiotica, 186:1-4, pp. 345394, 2011. Disponível em: http://www.jfsowa.com/pubs/egtut.pdf. Acesso em: 21 mar. 2016.

STAPLETON, G. A survey of reasoning systems based on Euler diagrams. In: Proceedings of the First International Workshop on Euler Diagrams, Electronic notes in Theoretical Computer Science, vol. 134, n. 1, pp. 127-151, 2005.

STRAWSON, P. F. Introduction to logical theory. London: Methuen \& Co Ltd, 1952. 